PALABRAS CLAVE I LIIMITE · URBANISMO CRÍTICO · CIUDAD CLANEA · EXCLUSIÓN · MORFOLOGÍA URBANA SPACIO SOCIAL

KEYWORDS | BOUNDARIES . CRITIC URBAN PIANNINE CONTEMPORARY CITY · EXCLUSION · URBAN MORPHOLOCY SOCIAL SPACE

JAVIERA MAIRA M.

\title{
Multidimensiones del límite y su renovación como concepto latente en la ciudad-contemporánea para un urbanismo crítico**
}

\section{INTRODUCCIÓN}

| RESUMEN |

Este artículo recupera y aproxima una redefinición al concepto de límite desde una mirada urbanaterritorial. Redefinir el límite es revisar sus definiciones desde distintos campos disciplinares para construir una propia definición que sirva para interpretar la ciudad por medio de sus estructuras o sistemas de límites. En la ciudad contemporánea coexiste una doble expresión del límite en la morfología urbana. Por un lado, los procesos de metropolización del territorio y explosión de la sociedad urbanizada de forma extendida y dispersa, han vuelto a la ciudad inabarcable. Por otro, la expresión de la idea original del límite-la delimitación, está expresada en el espacio social donde el conflicto social es un patrón cada vez más dominante que fortalece las divisiones del espacio urbano.

\footnotetext{
| ABSTRACT |

This paper attempts a redefinition of the concept of boundary from a territorial-urban view. Redefining a boundary involves reviewing its definitions from different disciplinary fields in order to build a own definition that serves to interpret the city through its structures or systems of limits. In the contemporary city a twin expression of boundaries exist in urban morphology. On one hand, the processes of planning and metropolization and the explosion of urbanized society that is widespread and dispersed, and which has reverted the city to a state of incomprehensibility. On the other hand, there is the original idea of the division-limit, expressed in the social space where social conflict is an increasingly dominant pattern that strengthens the divisions of urban space.
}

Pensar la disciplina urbanística como acción crítica es hoy tan aguda como en otros momentos de crisis, sobre todo aquellos que la vieron nacer, pero quizá hoy es uno de los momentos donde esta condición se convierte en acción y se torna vigente, transformándola en una posición que se debe adoptar urgentemente. La crisis del capitalismo, ilustrada en la explosión de la burbuja inmobiliaria como práctica del sistema financiero que azota en estos momentos a España, no es más que el reflejo de una crónica de una muerte anunciada.

Este desgaste recoge la idea de límite-limitación, como fin de algo, de un modelo, reflejando una ruptura o punto de inflexión. Las limitaciones estarían determinadas por el sistema de consumo o gasto, traducido a un hiperconsumo que generó tal nivel de riquezas globales que se pensaron ilimitadas y que ha convertido al mundo en un jolgorio desarrollista, como si de una gran fiesta se tratara, dejando a pocos indiferentes, convirtiéndose en la expresión fiel de la era del progreso de la posmodernidad.
Esta trajo consigo toda una gama de pensadores críticos que nos hacen reflexionar sobre nuestra condición y propia existencia, en la que se ha abandonado al hombre medio, reemplazando al sujeto por el objeto.

La ciudad, al ser el hábitat natural de la sociedad, es la que por siempre sirve de sustento para su desenvolvimiento y en ella surge la disciplina urbanística con objeto de remediar los males que en ella emergen, intentando corregir las deficiencias que el propio sistema urbano produce con el surgimiento de la ciudad industrial (Benévolo, 1992; Hall, 1996). La ciudad contemporánea ha dejado de manifiesto las limitaciones de la propia disciplina en el cabal cumplimiento de sus objetivos. La crisis del planeamiento actual se caracteriza por haber sido presa de la dominación del liberalismo y los procesos de desregulación, debilitando la disciplina urbanística y a la vez posicionando el desarrollo urbano.

Esta actitud crítica ha hecho visible situaciones de cansancio, frustración, desolación, desamparo, en los cuales los recientes movimientos de revueltas han recuperado

* Arquitecta. Dra (c) U.P.M. (Área del conocimiento Urbanística y Ordenación del Territorio). Académica Instituto Arquitectura y Urbanismo, Universidad Austral de Chile.

** Este artículo expone resultados preliminares de la tesis doctoral denominada «Redefinición del concepto de límite. Límites y su papel en la reproducción social. El caso de la ciudad de Valdivia» (defensa en 2013). 
1. Ocupación de la calle como espacio ciudadano para la manifestación de los movimientos de revuelta. a: ocupación Puerta del Sol, movimiento 15M en Presentación de Erik Swyngedouws (Fuente: http://www.thepolisblog. org/2011/06/eric-swyngedouw-on-designing-post.html).

b: ocupación en Londres, créditos de Wasi Daniju (Fuente: http://www.thepolisblog.org/201 1/10/london-protestersturn-public-space.html).
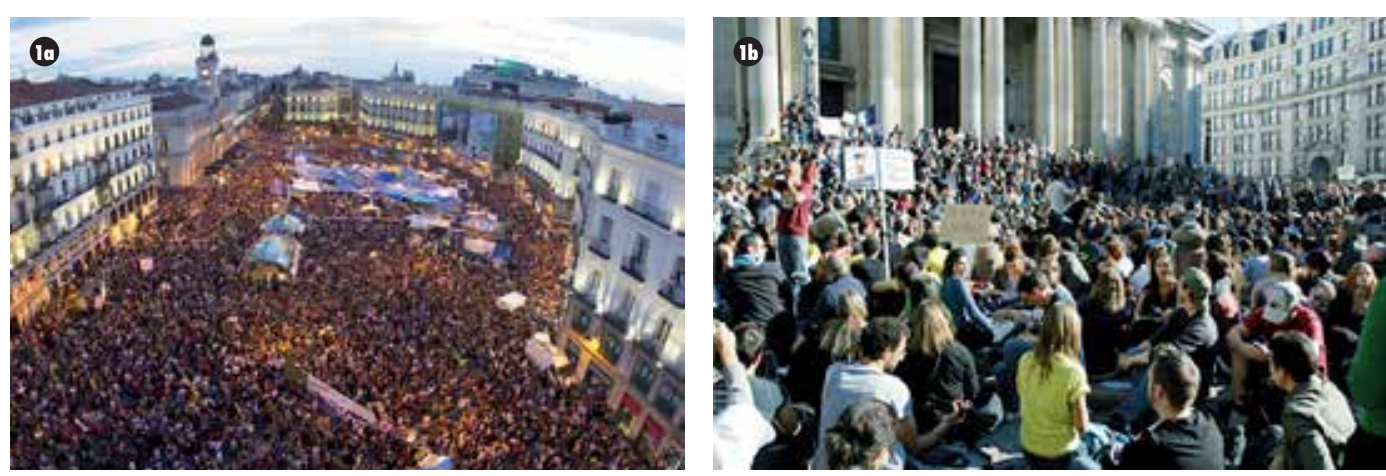

a la calle, como su espacio por esencia de libre expresión, el que sirve para expresar su condición ciudadana, muchas veces olvidada, reclamando en este punto de inflexión, su derecho al espacio urbano. Esto ha centrado la atención y el debate de muchos prestigiosos investigadores de ojo crítico sobre los procesos sociales en la ciudad. Nos sirven de ejemplo local nuestro movimiento estudiantil, y de referencia internacional el nacimiento de los llamados «nuevos espacios de insurgencia» ${ }^{[1]}$, como ocupación del espacio urbano de forma contestataria: el movimiento de los indignados $15 \mathrm{M}$ en la puerta del Sol en Madrid y los ocupas en Wall Street en New York. Estos han centrado la atención de un debate actual de la ciudad como construcción social, el que ha sido eje del pensamiento crítico en el campo que nos ocupa, el espacio urbano (Harvey, 2008; Mitchell, 2012; Marcuse, 2011; Lubin, 2012). Aunque enérgicos y renovados, los movimientos urbanos no han logrado sabotear el muro robusto y protegido de la hegemonía política, económica capitalista o más recientemente llamada inmobiliaria (Roch, 2008; Sabatini 2009).

Se explora por tanto el concepto de límite desde una mirada crítica, producto de su reconocimiento en cada vez más escenarios urbanos. Los límites se manifiestan como eminentemente sociales, expresando siempre en su sustrato formas de conflicto social, aunque además sean naturales, artificiales, perceptuales, jurídicas, etcétera.

Hay ciertas nociones del límite que han acompañado la historia de la arquitectura y la ciudad, otorgando significancia y contención al espacio, principalmente como elemento físico del espacio construido. Delimitamos para dotar de significado, así como compartimentamos el saber para su mejor comprensión, dividimos nuestro quehacer para así establecer el reconocimiento de las cosas, pero lo que pone en relación las partes, será una especie de límite cuyo papel es intermediar para a su vez dotarlas de significado (Simmel, 1986). Este espacio de transición entre dos situaciones disímiles, es en el cual se sitúa la noción de límite que aquí desarrollamos, que busca interpretar la ciudad a través de sus límites y demostrarlo como un elemento estructurador determinante de la morfología de la ciudad.

Lo que advertimos, repensando el concepto de límite en la ciudad contemporánea, es que este ya no solo se puede entender asociado a la configuración del espacio físico, simbólico, políitico y jurídico, estableciendo las reglas de lo urbano en un terreno delimitado (Weber, 1987), sino también como la manifestación

[1] N. del T. «New spaces of insugency» corresponde al nombre de un número especial editado el año 2011 por la revista The Berkeley PlanNIng a propósito del movimiento ocupa en Nueva York, el que evidenció la transformación del espacio cuando este se ocupa como espacio para la representación, lo que obliga a defenderlo con máxima convicción.

de una fractura en el espacio, entendido como lugar contenedor y «dador» de cualidad urbana. Actualmente predomina un entendimiento del límite más bien como expresión de fractura del espacio que como contenedor.

Este artículo recupera y aproxima una redefinición al concepto de límite desde una mirada urbana-territorial. Redefinir el límite es revisar sus definiciones desde distintos campos disciplinares (filosofía, geografía, etc.), para construir una propia definición que sirva para interpretar la ciudad por medio de sus estructuras o sistemas de límites.

\section{LOS LÍMITES COMO MODELADORES DE LA MORFOLOGÍA URBANA}

El cambio de siglo y el rápido consumo que han transformado social, política y culturalmente a nuestro mundo poseen como rasgo común que persiste el deseo de control de los sucesos. La vida moderna ha fortalecido el conocimiento de la realidad, en tanto mejorado las formas de predictibilidad ante la impredictibilidad. Uno de los conceptos enraizados en la concepción de ciudad es el de seguridad, en contraposición al miedo. El control mediante el dominio es la herramienta que posibilita la superación del peligro.

La evolución de la idea ancestral acuñada en el mito de la ciudad de Caín, como búsqueda de protección del miedo amenazador, donde el hogar es la reducción del mito del refugio, posiciona el funcionamiento de la ciudad como refugio en la medida que logra ser comprendida, permitido en ese entonces por la presencia de unos límites dentro de los cuales la ciudad puede ser abarcable intelectualmente. Esta facultad interpretativa del ser humano se logra mediante el control, el cual se expresa en los diferentes estadios de la historia de la ciudad, con distintas técnicas o instrumentos de representatividad; desde la pintura hasta el movimiento introducido con el cine la ciudad siempre busca ser representada (de Azúa, 1999). 
En la ciudad contemporánea coexiste una doble expresión del límite en la morfología urbana. Por un lado, los procesos de metropolización del territorio y explosión de la sociedad urbanizada de forma extendida y dispersa, han vuelto a la ciudad inabarcable y materialmente impresentable; se utilizan como expresión para estos fenómenos el término borderless, donde los límites se han perdido, desapareciendo en el contexto de la globalización (Newman, 2007). Por otro, la expresión de la idea original del límite-la delimitación, está expresada en el espacio social (urbano). Bajo la persistencia de la necesidad de control en la construcción de la ciudad y sus modelos de convivencia actual, alimentada por la política del miedo, es que surgen como manifestación de esta obsesión de control de los procesos urbanos nuevos fragmentos, bajo algunas contemporáneas directrices del actual urbanismo que reproducen verdaderos simulacros, erguidos bajo directrices de planificación que determinan las conocidas comunidades cerradas con estrictos, o no tanto, códigos que las rigen. Se constata la permanencia de una función delimitadora de las estructuras de límite para dotarla de su significado, pero en estos casos, para conseguir tal tipo de ciudad se hace necesario su cerramiento, empaquetamiento o aislamiento, la que resulta en apariencia rica en diversidad, pero alejada de sus inconvenientes, de lo real, entonces para que el modelo funcione con éxito se quitan de la vista los aspectos negativos de la realidad, consiguiendo fragmentos «urbanoides» (Goldenberg), explotando o generalizando la exclusión y la segregación. Desde esta perspectiva, el término bounding, es más explicativo del fenómeno que nos interesa estudiar, que dice relación con la naturaleza de la delimitación fundada en la compartimentación o demarcación de grupos o personas que se encierran en compartimentos sociales y espaciales, estableciendo jerarquías en las relaciones de los límites influenciadas por la percepción, la que designa el valor de uso del espacio por sobre su jurisdicción (Newman y Paasi, 1998).

En línea con la historia del orden impuesto frente al caos vigente aún en la ciudad

Evolución límites en la historia urbana preindustrial

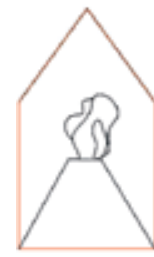

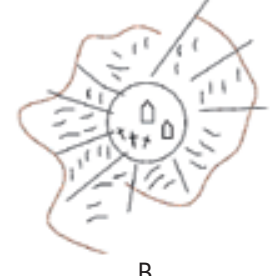

B

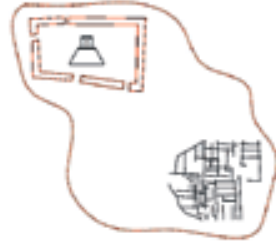

C

Leyenda

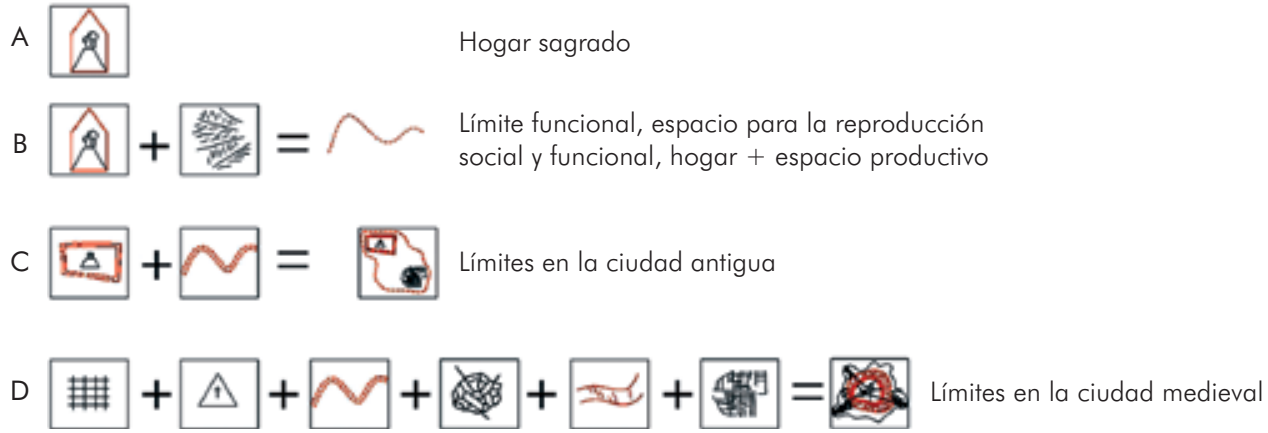

moderna, aunque posteriormente rechazado o reinventado, lo que resulta es que la ciudad es posible de entenderse integrada por partes, que principalmente responden a definiciones de uso y criterios para su funcionalidad. Sin embargo, hay otro orden de particiones al interior de la ciudad, que refuerzan las relaciones sociales que produce su propio funcionamiento, que son reflejo de una consecuencia jerárquica de una organización de funciones, desde un prisma eminentemente social la manifestación de las distintas relaciones sociales de una organización económica de la ciudad (Marcuse, 1994).

El conflicto social en la ciudad contemporánea es un patrón cada vez más dominante, esta tendencia creciente es cada vez más acusada, aunque a veces oculta, las partes suelen estar ordenados en el espacio por relaciones jerárquicas de poder y riqueza de los residentes, aunque dependan de fuerzas superiores y sean interdependientes entre sí (ldib). La desigualdad en el espacio urbano surge como un resultado persistente de un modelo en crisis, es decir, de un modelo de sociedad. El indicador más gráfico de esta expresión es el aumento exponencial de las diferencias de renta, de 16 veces entre lo más bajo y lo más alto, se ha pasado a $300 \mathrm{e}$ incluso a 3.000 veces, esto no solo entre países ricos y pobres, si no sobre todo al interior de las mismas ciudades, ilustrado recientemente en los países de economía emergentes como China e India, aunque generalizado el aumento de la brecha entre ricos y pobres ha alcanzado su nivel más alto en los últimos 50 años (OCDE, $2011)$.

Los límites, por su carácter separador, se expresan en líneas o bordes (visibles como invisibles) que encierran personas y determinan espacios, e inciden directamente en la extensión de la inclusión y exclusión de los miembros del mismo grupo (Sibley, 1996). Los límites al interior de la ciudad, urbanos, serán instrumentos imprescindibles para configurar la forma social y la forma urbana, modelando lo equivalente, lo similar, lo que funciona como un todo orgánico, como asimismo la exclusión y la segregación, levantando de igual modo mecanismos de seguridad construidos o preceptuales, que protagonizan la forma urbana y la morfología del espacio social. 
3. Mumbai, las dos caras de la misma ciudad, crecimiento y desigualdad (Fuente: Google [http://www.mumbailocal. net/2938/dharavi-slum-mumbai/]; Google [http://uraniumdiaries.blogspot.com/2009/06/las-12-ciudades-masgrandes-del-mundo.html]).

\section{ASPECTOS CONSTITUYENTES PARA UNA REDEFINICIÓN DEL LÍMITE}

El término límite posee variadas acepciones como también ha tenido muchas formas en la historia. Las ciudades han estado ligadas desde sus inicios a estructuras de límites que sirven para ir desvelando los objetos particulares de cada momento y situación. Por otro lado, para una mejor comprensión de sus manifestaciones en el espacio social, físico y cultural, requiere nutrir su estudio desde otros campos del conocimiento que permiten recoger aspectos constituyentes para una aproximación teórica.

Si bien, asociado al término desde la semántica se identifican cuatro categorías: magnitud, espacio, situación y función, es desde su raíz etimológica donde queda más claro uno de los aspectos sustanciales de su significado. El término Limes (lîmitis), entendido como frontera, se le denominaba «a la franja de territorio habitada y colonizada por los limitanei o habitantes del limes» ${ }^{[2]}$ (Trias, 1995), hace distinguible la dualidad entre la idea de frontera como línea y límite como área contenedora. Es la segunda noción la que sirve para visualizar el límite como un espacio-umbral habitado y la que enlaza con la dimensión urbana y territorial que nos atañe.

Destacan algunas de sus expresiones más utilizadas en las lenguas inglesa y alemana. El término principal común que aparece en las lenguas inglesa y alemana es borde y en segundo lugar frontera, expresados como Boundary y die Grenze, aludiendo ambos a un carácter espacial. Desde la filosofía y su profunda tradición alemana, el término usado es die grezne, el cual Hegel utilizaba para referirse al estudio del límite en sus escritos y reflexiones, definido en su relación antagónica como unión de algo y su negación. Boundaries, por su parte, es el término utilizado desde el mundo anglosajón principalmente usado en geografía, donde
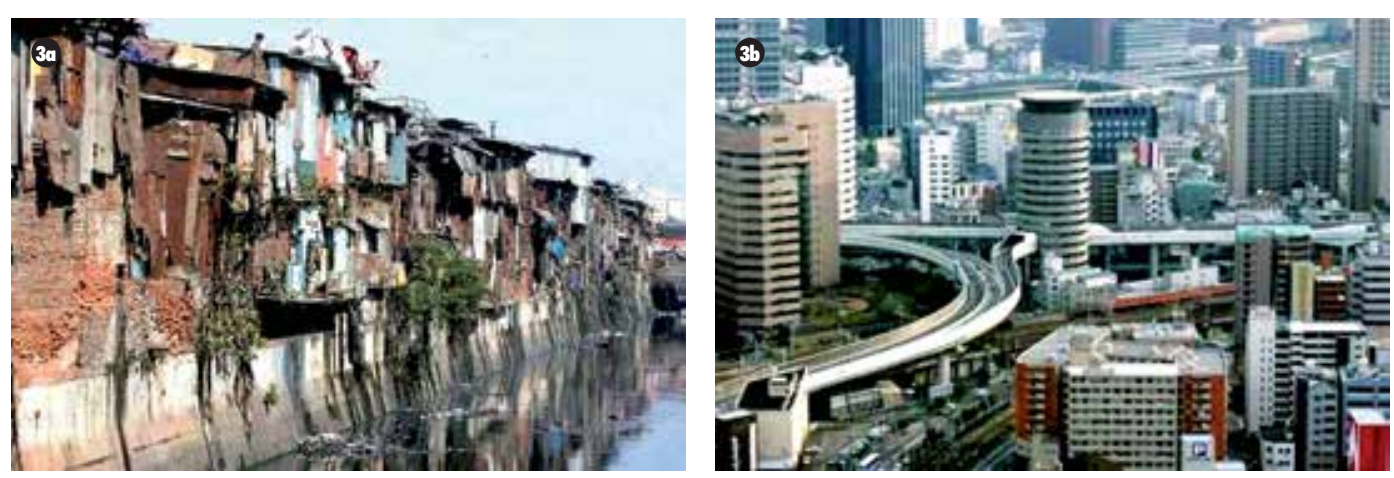

aparece como temática emergente el concepto de bounding-delimitación a propósito de nuevas aproximaciones de límite y entendido como fenómeno resultante de la idea de compartimentación.

La revisión de la evolución del límite en la ciudad es la revisión de la historia del conflicto, tanto desde una perspectiva marxista, con énfasis en la dimensión económica de la ciudad, la división del trabajo y la consecuente lucha de clases, como de los conflictos que estarían producidos por modos de producción y las consecutivas relaciones de reproducción inherentes desde la ciudad industrial.

Por otro lado, el permanente conflicto entre el hombre y naturaleza permite establecer relaciones y estructuras de límites desde la oposición campo-ciudad a la dialéctica actual, que más bien puede mencionarse entre urbanismo y ecología (medio ambiente). Esta perspectiva estudia los hechos como sucesos en el tiempo que permitan conocer la sociedad que determina a su vez las estructuras de límite.

Al delimitar un espacio le conferimos carácter y legibilidad, se hace reconocible, pero al mismo tiempo se confiere estigmatización, separando las partes de la ciudad que deben funcionar integradas. Esto no es condición del espacio urbano-público, teatro de la expresión colectiva.
Sin embargo, en la determinación del lugar, desde su propia especialidad, si su carácter está determinado por el tratamiento concreto de los elementos que lo delimitan, los bordes adquieren un rol estructurador en la conformación del espacio concebido como lugar, con una naturaleza especifica y concreta de sus límites. Están presentes para otorgar orientación, en tanto estructuras existentes que delimitan la percepción, o frontera física, permeable, palpable, nítidamente perceptible. Los límites artificiales encierran dentro de sí algunos casos la geografía (social) del lugar, no tanto así los límites naturales, que aunque fronteras muchas veces, sirven más bien para delimitar algo inabarcable.

Los aspectos identificados sirven para poner a punto y depurar el concepto de límite, es posible otorgarle su dimensión plural, o naturaleza constituyente del fenómeno urbano. Aparecen en sus manifestaciones, características como el conflicto, formas históricas de gestionarse, desde formas de dominación o colaboración, orientando el orden social y sus modos de reproducción y representación en los diferentes dominios.

La Tabla 1, corresponde a una primera clasificación de los elementos presentes que verifican su carácter multidimensional, han sido ordenados en los distintos tipos, dimensiones, manifestaciones, características, función y fenómenos en relación. estructuras características determinando en 
4. Mapa mental que ilustra relaciones establecidas entre los términos asociados a la palabra e idea de límite, según la revisión desde diferentes campos disciplinares (Fuente: Elaboración propia).

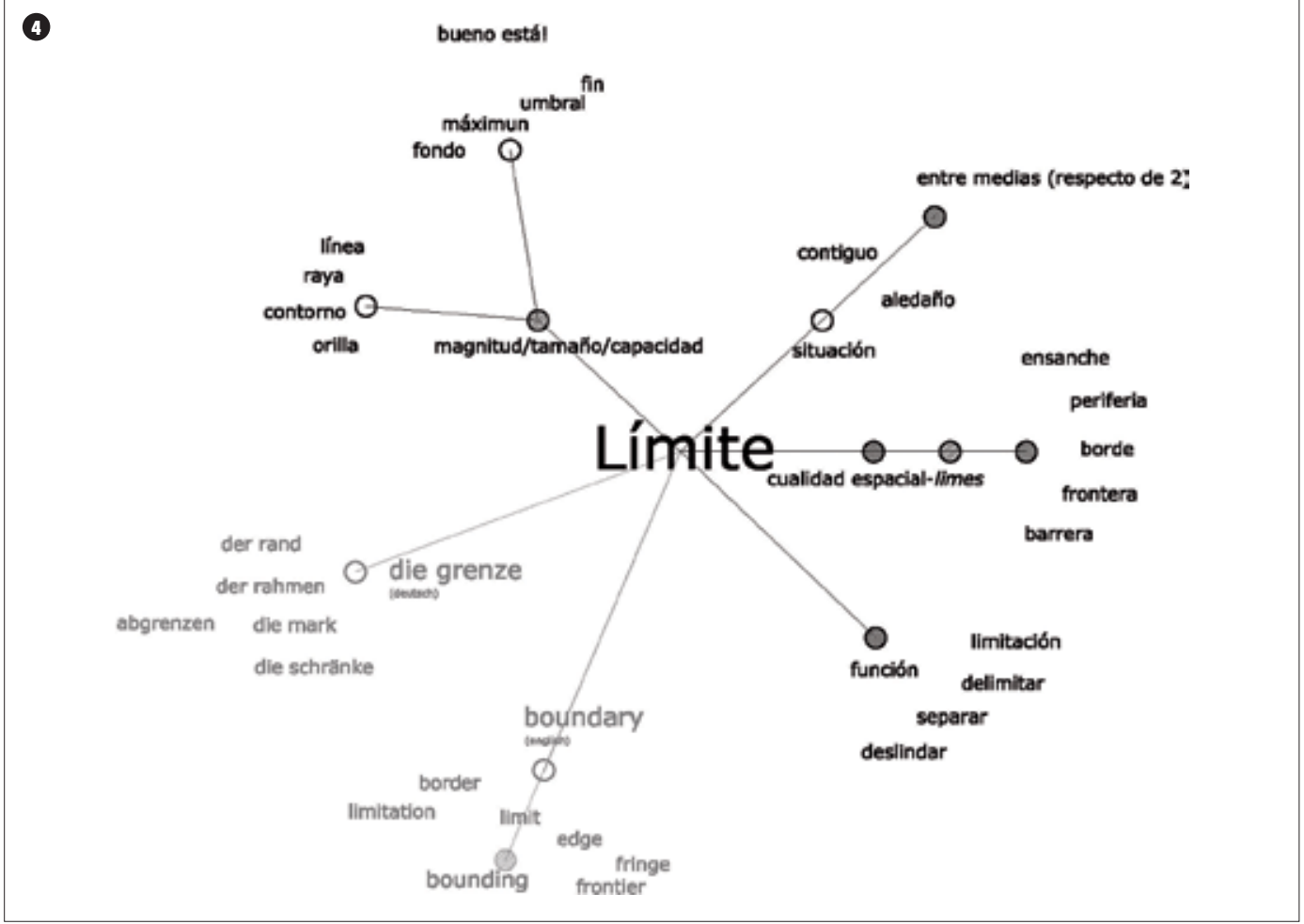

\section{LA MULTIDIMENSIONALIDAD DEL LÍMITE}

Ciertas características del fenómeno urbano, son a su vez constituyentes de la existencia de límites en la ciudad, se exploran mediante la identificación de su espacio social, funcional, urbano, que subyace o que los ha determinado.

El lugar del límite, es un lugar producto de cierto tipo de relaciones sociales, relaciones de producción del espacio, un espacio de dominación, conflicto, enfrentamiento, choque de situaciones antagónicas (que no median entre sí), pero que se enfrentan en este espacio umbral que los pone en relación.

Al estudiar el límite desde los distintos campos del conocimiento hay lecturas paralelas, que al ir entresacando tanto de la historia urbana como de la historia social, es posible entretejer así la historia de un conflicto en el espacio urbano.

TABLA 1

Elementos para la elaboración de una Taxonomía a partir de los fenómenos asociados al Límite en la ciudad (Fuente: elaboración propia)

\begin{tabular}{|c|c|c|c|c|c|c|}
\hline Tipos & Dimensiones & Manifestaciones/Expresión & Características & Función/Rol & En relación a & Fenómenos \\
\hline Natural & Límite y espacio social & Social & Adaptabilidad & Conserva condición de reproducción & Crecimiento & Demográficos \\
\hline Artificial & & Material & Permanencia & Control & Control & Político \\
\hline Material & Límite y memoria & Funcional & Transformación & Regulación & & Económico \\
\hline Construido & perceptual & & Permeabilidad & Protección & & \\
\hline Simbólico & & Física & & & & \\
\hline Sagrado & Límite y manifestación & & Estructural & & & Dominación \\
\hline Funcional & material & & Instrumental & & & Exclusión \\
\hline \multirow[t]{2}{*}{ Jurídico } & social & & & & & Segregación \\
\hline & instrumental & & Atemporal & & & \\
\hline \multirow[t]{9}{*}{ Social } & & & Transcultural & & & \\
\hline & Límites y permanencias & & Espacial & & & \\
\hline & espacio/temporal & & & & & \\
\hline & (dimensión evolutiva) & & & & & \\
\hline & Límite y figuraciones & & & & & \\
\hline & material & & & & & \\
\hline & elementos & & & & & \\
\hline & Límite y paisaje & & & & & \\
\hline & ecología & & & & & \\
\hline
\end{tabular}


Sin embargo, es posible establecer que el límite es de naturaleza social. El límite no es una línea, si no más bien un espacio habitado que posee medidas, altura, dimensiones, cualidades y funciones. Es particular.

Lo multidimensional constituye uno de los aspectos para su definición y la multiescalaridad su expresión. El límite se expresa cada vez más en el espacio urbano, pero también se reconoce en el espacio arquitectónico, en el territorio y con una nueva mirada se expresa en el paisaje constituyendo su transformación.

Hay sucesivas especies de límite, que a su vez son bordes y frontera, son espacios límite - de borde-frontera.

\section{REFLEXIONES FINALES PARA UN URBANISMO CRÍTICO}

El estudio de las manifestaciones del Límite en la ciudad y su papel estructurante en la morfología urbana, relevan una mirada crítica, la cual debe atravesar la práctica del urbanismo de forma permanente, para no olvidar la sustancia política de las ciudades urbanismo en tanto, política pública. La dimensión social de la ciudad las hace políticas y las relaciona a diversas esferas de poder.

Los límites son estructuras que permiten las condiciones de existencia de un determinado sistema social, el límite se entiende así como una construcción social.

El límite posee un valor estructural, constituyente del fenómeno urbano desde los estudios de morfología urbana bajo el enfoque de los procesos sociales, en esa mirada defendemos que el espacio es socialmente producido.

Contribuciones para el cambio de rumbo de la urbanística son necesarias y urgentes. En este escenario nos corresponde como estudiosos de los fenómenos sociales, hacerlas al alero de una disciplina que nos acoge, entendiendo la morfología urbana como un espacio de vinculación entre la organización de los grupos sociales y su realidad material, su sustrato físico, asumiendo que ambas instancias tienen modalidades propias, son construcciones sociales y ambas evolucionan.

\section{REFERENCIAS BIBLIOGRÁFICAS}

Azúa de, F. La invención de Caín. Ciudades y ciudadanos. Madrid: Alfaguara, 1999.

Benévolo, L. Orígenes del urbanismo moderno. Madrid: Celeste, 1992.

Hall, P. Ciudades del mañana. Historia del urbanismo en el siglo XX. Barcelona: Ediciones del Serbal, 1996

Harvey, D. «The right to the city». New Left Review 2008; 53 [fecha de acceso 23 abril 2012 en: http://newlwftreview.org/Pview=2740].

Lubin, J. «The "Occupy" Movement: emerging protest forms and contested urban spaces». Berkeley Planning Journal 2012; 25(1): 184-97. UC Berkeley [fecha de acceso 16 agosto 2012 en: http://escholarship.org/uc/item/5rb320n3].

Marcuse, P. «Not chaos but walls: postmodernism and partitioned city». En: Watson, E. y Gibson, K. Postmodern cities and spaces. Cambridge: Blackwell Publishing, 1994; 243-54.

Marcuse, P. «Occupy and the provision of public space: the city's responsibilities». Blog de Peter Marcuse,
01 de diciembre de 2011 [fecha de acceso 10 marzo 2012 en: http://pmarcuse.wordpress. com/2011/12/01/occupy-and-the-provision-ofpublic-space-the-citys-responsibility].

Mitchell, D. «Revueltas: la otra globalización urbana». Urban NSO3 (Madrid) 2012; 11 -19

Newman, D. «Boundaries». En: Agnew, J., Mitchell, K., Tuathail G. y Toal G. A companion to political geography. Cambridge: Blackwell Publishing, 2007; 9: 123-37.

Newman, D. y Paasi, A. «Fences and neighbours in the postmodern world: boundary narratives in politica geography». Progress in Human Geography 1998; 22(2): 186-207.

OECD. Divided we stand. Why inequality keeps rising. Special focus: Inequality in Emerging Economies (EEs), 2011 [en: http://www. oecd.org/els/socialpoliciesanddata/ incomedistributionand poverty.htm].

Roch, F. «La deriva patológica del espacio social en el modelo inmobiliario neoliberal madrileño». Diez años de cambios en el Mundo, en la Geografía y en las Ciencias Sociales, 1999-2008. Actas del X Coloquio Internacional de Geocrítica, 26-30 mayo. Barcelona: Universidad de Barcelona, 2008

Sabatini, F. «Gentrificación de la periferia popular en la ciudad latinoamericana: fuerza de transformación y espacio de acción». En: FAU, Universidad de Chile. Ciudad y deseo. Exclusión y diversidad: del barrio a la metrópolis. Santiago, Chile: Universidad de Chile, 2009: 56-59.

Sibley, D. Geographies of exclusion: society and difference in the west. Londres: Routledge, 1996.

Simmel, G. Puente y puerta. El individuo y la libertad. Barcelona: Península, 1986; 29-34

Trías, E. La razón fronteriza. Barcelona: Destino, 1999

Weber, M. La ciudad. Madrid: La Piqueta, 1987 (original, Die Stadt, 1925). 